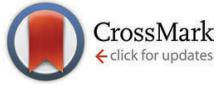

Cite this: Mol. BioSyst., 2015, 11,317

DOI: $10.1039 / \mathrm{c} 4 \mathrm{mb} 90037 \mathrm{e}$

www.rsc.org/molecularbiosystems

\section{Correction: Rapidly improved determination of metabolites from biological data sets using the high-efficient TransOmics tool}

\author{
Aihua Zhang, ${ }^{a}$ Xiaohang Zhou, ${ }^{a}$ Hongwei Zhao, ${ }^{\text {b }}$ Yu Guan, ${ }^{a}$ Shiyu Zou, ${ }^{\text {b }}$ \\ Guang-li Yan, ${ }^{a}$ Chung Wah Ma, ${ }^{b}$ Qi Liu ${ }^{a}$ and Xijun Wang*a
}

Correction for 'Rapidly improved determination of metabolites from biological data sets using the high-efficient TransOmics tool' by Aihua Zhang et al., Mol. BioSyst., 2014, 10, 2160-2165.

The spellings of two of the authors' names were incorrect. Zhonghua Ma should read Chung Wah Ma and Shiyu Zhou should read Shiyu Zou. The full author list should be Aihua Zhang ${ }^{\mathrm{a}}$, Xiaohang Zhou ${ }^{\mathrm{a}}$, Hongwei Zhao ${ }^{\mathrm{b}}$, Yu Guan ${ }^{\mathrm{a}}$, Shiyu Zou ${ }^{\mathrm{b}}$, Guang-li Yanª Chung Wah Ma ${ }^{\mathrm{b}}$, Qi Liu ${ }^{\mathrm{a}}$, Xijun Wang. ${ }^{{ }^{*}}$

The Royal Society of Chemistry apologises for these errors and any consequent inconvenience to authors and readers.

${ }^{a}$ National TCM Key Laboratory of Serum Pharmacochemistry, Key Laboratory of Metabolomics and Chinmedomics, Department of Pharmaceutical Analysis, Heilongjiang University of Chinese Medicine, Heping Road 24, Harbin 150040, China. E-mail: aihuaz@yeah.net, xijunwangls@126.com; Fax: +86-451-82193038; Tel: $+86-451-82193038$

${ }^{b}$ Infinitus (China) Company Ltd, Guangzhou, China 